\title{
A!
}

This is an electronic reprint of the original article.

This reprint may differ from the original in pagination and typographic detail.

Östergård, Patric R.J.; Pöllänen, Antti

\section{New Results on Tripod Packings}

Published in:

Discrete and Computational Geometry

DOI:

$10.1007 / \mathrm{s} 00454-018-0012-2$

Published: 15/03/2019

Document Version

Peer reviewed version

Please cite the original version:

Östergård, P. R. J., \& Pöllänen, A. (2019). New Results on Tripod Packings. Discrete and Computational Geometry, 61(2), 271-284. https://doi.org/10.1007/s00454-018-0012-2

This material is protected by copyright and other intellectual property rights, and duplication or sale of all or part of any of the repository collections is not permitted, except that material may be duplicated by you for your research use or educational purposes in electronic or print form. You must obtain permission for any other use. Electronic or print copies may not be offered, whether for sale or otherwise to anyone who is not an authorised user. 


\title{
New Results on Tripod Packings*
}

\author{
Patric R. J. Östergård and Antti Pöllänen \\ Department of Communications and Networking \\ Aalto University School of Electrical Engineering \\ P.O. Box 15400, 00076 Aalto, Finland \\ \{patric.ostergard, antti.pollanen\}@aalto.fi
}

\begin{abstract}
Consider an $n \times n \times n$ cube $Q$ consisting of $n^{3}$ unit cubes. A tripod of order $n$ is obtained by taking the $3 n-2$ unit cubes along three mutually adjacent edges of $Q$. The unit cube corresponding to the vertex of $Q$ where the edges meet is called the center cube of the tripod. The function $f(n)$ is defined as the largest number of integral translates of such a tripod that have disjoint interiors and whose center cubes coincide with unit cubes of $Q$. The value of $f(n)$ has earlier been determined for $n \leq 9$.

The function $f(n)$ is here studied in the framework of the maximum clique problem, and the values $f(10)=32$ and $f(11)=38$ are obtained computationally. Moreover, by prescribing symmetries, constructive lower bounds on $f(n)$ are obtained for $n \leq 26$. A conjecture that $f(n)$ is always attained by a packing with a symmetry of order 3 that rotates $Q$ around the axis through two opposite vertices is disproved.
\end{abstract}

Keywords: clique, monotonic matrix, packing, semicross, Stein corner, tripod

MSC Primary: 52C17

\section{Introduction}

Solomon Golomb [2] gave a general definition of coding distance functions for various types of error-correcting codes, unifying various distance functions including those introduced by Hamming, Lee, Shannon, and Stein. (Note

*Supported in part by the Academy of Finland, Project \#289002. 
that not all of these functions are metrics.) The problem of maximizing the rate of a code for a given distance function and error-correcting capability can be phrased as that of finding a maximum packing, where a packing is a set of error spheres with disjoint interiors.

One type of error spheres defined by Golomb was introduced even earlier by Stein [9] and is known as a Stein corner (or semicross). In his seminal paper [9], Stein studied packings of such spheres in an algebra framework. These packings can be studied in Euclidean spaces or in (finite) parts thereof. In the finite case, which is considered here, the spheres and their variants are related to error models for systems with asymmetric limited-magnitude errors [1], which are relevant, for example, for certain flash memories [5]. Lattice packings in Euclidean spaces using Stein corners are currently well understood [11], but arbitrary packings achieve decisively higher densities $[3,10]$.

In the 3-dimensional case, the smallest nontrivial case, a Stein corner is known as a tripod. Specifically, consider an $n \times n \times n$ cube $Q$ consisting of $n^{3}$ unit cubes. A tripod of order $n$ is obtained by taking the $3 n-2$ unit cubes along three mutually adjacent edges of $Q$. The unit cube corresponding to the vertex of $Q$ where the edges meet is called the center cube of the tripod. In the study of maximum packings, we define $f(n)$ as the largest number of integral translates of such a tripod that have disjoint interiors and whose center cubes coincide with unit cubes of $Q$. A tripod of order 4 is depicted in Figure 1.



Figure 1: A tripod of order 4 (center cube not visible) 
The value of $f(n)$ has earlier been determined for $n \leq 9$ in work by Joy $(1 \leq n \leq 5)$, Tiskin $(n=6)$, and Szabó $(7 \leq n \leq 9)$; see [11], [13], and [12], respectively. Asymptotically, we have a lower bound on $f(n)$ of $\Omega\left(n^{1.534}\right)$ and an upper bound of $O\left(n^{2} /(\log n)^{1 / 15}\right)[13]$.

Computational techniques have played a central role in earlier work. This work is here continued, and it is among other things shown that $f(10)=32$ and $f(11)=38$. Moreover, by prescribing symmetries, constructive lower bounds on $f(n)$ are obtained for $n \leq 26$. The old and new results-lower bounds and exact values for $f(n)$-are summarized in Table 1.

Table 1: Old and new results on $f(n)$

\begin{tabular}{|c|c|c|c|c|c|c|c|}
\hline$n$ & Old & New & $n$ & Old & New & $\begin{array}{ll}n & \text { Old }\end{array}$ & New \\
\hline 1 & 1 & & 10 & $>32$ & 32 & 19 & $>87$ \\
\hline 2 & 2 & & 11 & $\geq 37$ & 38 & 20 & $\geq 94$ \\
\hline 3 & 5 & & 12 & $\geq 43$ & & 21 & $\geq 104$ \\
\hline 4 & 8 & & 13 & $\geq 49$ & & 22 & $\geq 110$ \\
\hline 5 & 11 & & 14 & $\geq 55$ & & 23 & $\geq 121$ \\
\hline 6 & 14 & & 15 & $\geq 61$ & & 24 & $\geq 126$ \\
\hline 7 & 19 & & 16 & $\geq 65$ & $\geq 68$ & 25 & $\geq 136$ \\
\hline 8 & 23 & & 17 & & $\geq 75$ & 26 & $\geq 144$ \\
\hline 9 & 28 & & 18 & & $\geq 80$ & & \\
\hline
\end{tabular}

In particular, the results for tripod packings with symmetries disprove a conjecture [13] that $f(n)$ is always attained by a packing with a symmetry of order 3 that rotates $Q$ around the axis through two opposite vertices.

The paper is organized as follows. In Section 2, various frameworks in which the current problem can be considered are discussed. A computational approach for determining $f(n)$ is considered in Section 3, with the cases of even and odd $n$ handled separately. The approach of prescribing symmetries of tripod packings is finally treated in Section 4.

\section{Three Frameworks}

To specify a translate of a tripod, it suffices to know the position of its center cube. Hence, a set of integral translates of a tripod with the center cubes within the big cube $Q$ can be described through a set of codewords in $Z_{n} \times Z_{n} \times Z_{n}$, where $Z_{n}=\{0,1, \ldots, n-1\}$. It is not difficult to check that the set of tripods is a packing if and only if each pair of codewords satisfies the 


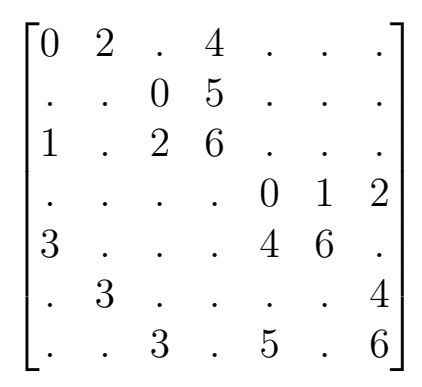

Figure 2: Optimal monotonic matrix for $n=7$

following conditions: (1) The codewords differ in at least two coordinates, and (2) If the codewords differ in exactly two coordinates, then one of the codewords must have a greater value in both of those coordinates. A code corresponding to a maximum packing is called optimal.

Example 1. The following code shows that $f(7) \geq 19$ : $\{000 ; 012,201,120$; $034,403,340 ; 135,513,351 ; 222 ; 236,623,362 ; 444 ; 456,645,564 ; 666\}$ (the partitioning of the codewords is for later purposes). By Table 1, this code is in fact optimal.

An alternative way of presenting such a code is through an $n \times n$ monotonic matrix. A monotonic matrix is a matrix with cells that are either empty or have one value from $Z_{n}$, such that the following conditions are satisfied:

(i) The values in a column are increasing from top to bottom.

(ii) The values in a row are increasing from left to right.

(iii) For two nonempty cells with the same value, the one below is also further on the right.

(Some authors display monotonic matrices in an alternative, equivalent way, where the values in a column are increasing from bottom to top; then also (iii) must be modified accordingly.) A monotonic matrix can be obtained directly from a code: for a codeword $\left(a_{1}, a_{2}, a_{3}\right)$, the value $a_{3}$ is given to the cell in row $a_{1}$ and column $a_{2}$ (numbering starts from 0 and goes from top to bottom and from left to right). Figure 2 depicts the $7 \times 7$ monotonic matrix obtained in this manner from the code in Example 1.

The described code and matrix frameworks both provide the same information about a tripod packing. We shall now look at a third framework, which is particularly useful when searching for a maximum packing. In such a search, it is essential to know whether two tripods have disjoint interiors or not. Then we may form a graph $G$ with $n^{3}$ vertices, one for each possible 
tripod, and with an edge between two tripods that have a disjoint interiors. Labeling the vertices with the corresponding codewords, there is an edge exactly when the conditions (1) and (2) for codes are satisfied.

Let $V(G)$ and $E(G)$ denote the sets of vertices and edges of the graph $G$, respectively. A clique in $G$ is a subset $C \subseteq V(G)$ such that $\{a, b\} \in E(G)$ whenever $a, b \in C$ and $a \neq b$. There is now a bijection between the cliques in $G$ and the sets of tripods with mutually disjoint interiors, so $f(n)$ equals the size of a largest clique in $G$, a maximum clique. In this paper, we will study $f(n)$ using computational techniques, utilizing the clique searching software Cliquer [6], which is based on [7].

To determine $f(n)$ we can do better than applying clique search directly to the graph $G$ defined above, and this is the topic of the next section. The approaches still consist of subtasks where all cliques or cliques of certain sizes are to be found in some precisely defined induced subgraph of $G$. Indeed, whenever a subtask consists of finding (typically all) tripod packings - or extensions of tripod packings - in a specific set, clique search is implicitly assumed. Specifically, when searching for extensions of a tripod packing $X \subseteq V(G)$ in $Y \subseteq V(G)$, we consider the subgraph of $G$ induced by

$$
\{y \in Y:\{x, y\} \in E(G) \text { for all } x \in X\} .
$$

Cliquer is a flexible piece of software that can handle the variants of clique search occurring here. In particular, the possibility of processing each clique further within a C program that calls Cliquer is very useful. See the user's guide [6] for details.

\section{Determining $f(n)$}

We use the following general approach to determine $f(n)$ for some given $n$. We consider the best known lower bound on $f(n)$, say $f(n) \geq s-1$, and carry out an exhaustive search for a tripod packing of size $s$. If there is no such packing, then we are done; otherwise the search is repeated for size $s+1$, and so on. Symmetries of tripod packings are discussed in the next section, whereafter the search algorithm is presented, and finally the results of the current work are listed.

\subsection{Symmetries of Tripod Packings}

Symmetries can be utilized in several ways in the study of tripod packings. First, symmetries of the ambient space speed up the search when determining $f(n)$ computationally. Second, symmetries can be prescribed for tripod 
packings when $n$ is so large that it is not feasible to determine $f(n)$ exactly; such packings still give constructive lower bounds on $f(n)$.

The big cube $Q$ has a symmetry group of order 48, but only 6 of those symmetries respect the way the tripods are directed. When viewing tripod packings as sets of codewords, these 6 symmetries are exactly the 3 ! permutations of the coordinates. However, when formulating the search problem in the framework of graphs and cliques, we may consider a symmetry group of size 12 rather than 6 . Namely, the mapping of codewords

$$
(x, y, z) \rightarrow(n-1-x, n-1-y, n-1-z)
$$

gives an automorphism of the graph $G$. (An automorphism of a graph $G$ is a bijection $f: V(G) \rightarrow V(G)$ such that for any $a, b \in V(G)$ we have $\{a, b\} \in E(G)$ if and only if $\{f(a), f(b)\} \in E(G)$.) The group of order 12, which we call $H$ in the sequel, can be generated by

$$
\begin{aligned}
& r(x, y, z)=(z, x, y) \\
& p(x, y, z)=(y, x, z), \text { and } \\
& o(x, y, z)=(n-1-x, n-1-y, n-1-z) .
\end{aligned}
$$

Denoting $(f \circ g)=f g$ and letting $\epsilon$ be the identity element, the set of elements of the group $H$ is

$$
\left\{\epsilon, r, r^{2}, o, o r, o r^{2}, p, p r, p r^{2}, o p, o p r, o p r^{2}\right\} .
$$

Note that $o$ commutes with every group element, so the group is isomorphic to the direct product of the group of order 2 and the symmetric group of degree 3 and order 6 . The group has the following subgroups, partitioned into conjugacy classes:

(a) $\langle\epsilon\rangle=\{\epsilon\}$,

(b) $\langle r\rangle=\left\{\epsilon, r, r^{2}\right\}$,

(c) $\langle o\rangle=\{\epsilon, o\}$,

(d) $\langle p\rangle=\{\epsilon, p\},\langle p r\rangle=\{\epsilon, p r\},\left\langle p r^{2}\right\rangle=\left\{\epsilon, p r^{2}\right\}$,

(e) $\langle r, o\rangle=\left\{\epsilon, r, r^{2}, o, o r, o r^{2}\right\}$,

(f) $\langle o p\rangle=\{\epsilon, o p\},\langle o p r\rangle=\{\epsilon, o p r\},\left\langle o p r^{2}\right\rangle=\left\{\epsilon, o p r^{2}\right\}$,

(g) $\langle r, p\rangle=\left\{\epsilon, r, r^{2}, p, p r, p r^{2}\right\}$,

(h) $\langle r, o p\rangle=\left\{\epsilon, r, r^{2}, o p, o p r, o p r^{2}\right\}$,

(i) $\langle o, p\rangle=\{\epsilon, o, p, o p\},\langle o, p r\rangle=\{\epsilon, o, p r, o p r\},\left\langle o, p r^{2}\right\rangle=\left\{\epsilon, o, p r^{2}, o p r^{2}\right\}$. 


\subsection{General Approach}

Before going into the details of the search algorithm, let us briefly elaborate on the role of symmetries in such a search.

The order of the group $H, 12$, is very small compared with orders of groups involved for many other types of construction problems in discrete mathematics [4], but the symmetries are still useful. The way this group is utilized here is one of the main differences between the current work and earlier studies. The approach to be described can directly be generalized to $k$-dimensional Stein corners; the order of the group, $2 k$ !, then grows rapidly with growing $k$.

The main idea behind utilizing symmetries is the following. Assume a group action where elements of the symmetry group act on tripods and sets of tripods in the obvious way. We say that two sets of tripods, $S$ and $T$ are isomorphic if $g S=T$ for some group element $g$ (formally, $S$ and $T$ are in the same orbit under the action of the given group). If $S$ and $T$ are isomorphic, then $g\left(S \cup S^{\prime}\right)=g S \cup g S^{\prime}=T \cup g S^{\prime}$, and every extension of $S$ is mapped by $g$ to an extension of $T$. Hence, in a situation like this it suffices to consider extensions of one of $S$ and $T$; the process of deleting isomorphic copies is termed isomorph rejection. We further call a group element $g$ for which $g S=S$ an automorphism of $S$; the set of all automorphisms of $S$ is called the automorphism group of $S$. A subgroup of the automorphism group is called a group of automorphisms.

One further idea is introduced in the current work. Actually, the idea is new only for this particular problem since it has frequently been used for other types of codes and combinatorial structures; see, for example, [4, Sect. 7.1.1]. Let $Q=P \cup R$ be a partition of the ambient space such that $P=g R$ for some element $g \in H$. Now we may without loss of generality assume that $|S \cap P| \geq|S \cap R|$ for the tripod packing $S$ that we construct, since $|S \cap P| \leq|S \cap R|$ implies that $|g S \cap P| \geq|g S \cap R|$.

We need consider the cases of even and odd $n$ separately, since the latter case requires a modification of the general idea. In the sequel, we always assume that $(0,0,0)$ and $(n-1, n-1, n-1)$ are in a packing; see [13, p. 1974] and [12, Lemma 2.1]. As these two words form an orbit under the action of $H$, they do not affect (techniques based on) the symmetries of the problem. 


\subsubsection{Even $n$}

For even $n$, we let

$$
\begin{aligned}
g & =o \\
P & =\{(x, y, z): 0 \leq x+y+z \leq 3 n / 2-2\} \\
R & =\{(x, y, z): 3 n / 2-1 \leq x+y+z \leq 3 n-3\}
\end{aligned}
$$

and when aiming at a tripod packing in $Q$ of size $s$, an intermediate goal of the search is to find, up to symmetry, the tripod packings in $P$ with size greater than or equal to $s / 2$.

The subgroup of $H$ that stabilizes $P$ contains only the permutations of the coordinates (in the coding framework), $\langle r, p\rangle$, and with a group of order 6 it is not necessary to use any advanced techniques for isomorph rejection [4]. Isomorph rejection amongst tripod packings in $P$ is conveniently carried out by defining a canonical form and accepting only packings that are in such a form. There are several ways of defining canonical forms; in the current work, two different approaches were used, one of which is the following.

Let us first recall the definition of the lexicographic order of $k$-tuples: for $A=\left(a_{1}, a_{2}, \ldots, a_{k}\right)$ and $B=\left(b_{1}, b_{2}, \ldots, b_{k}\right), A<B$ exactly when $a_{i}<$ $b_{i}$, where $i$ is the first entry where $a_{i} \neq b_{i}$. For our tailored approach of constructing packings, to be considered next, we need a different order: For two codewords $A$ and $B$ with coordinate values in $Z_{n}, A<B$ exactly when $\sum_{i=1}^{k} a_{i}<\sum_{i=1}^{k} b_{i}$; or $\sum_{i=1}^{k} a_{i}=\sum_{i=1}^{k} b_{i}$ and $A$ is lexicographically smaller than $B$ (here $k=3$ ). The order of codewords in turn induces an order on sets of codewords: In each set, sort the codewords in increasing order and use lexicographic order for final comparison. We now define the canonical form of a tripod packing as the packing in its orbit that is smallest with the defined order.

Since we want to start carrying out isomorph rejection as early as possible, we go to packings in $P$ via an intermediate step. For the current problem, such an intermediate step has a minor impact on the performance, but it is very useful for parallelization purposes. Specifically, we first find all tripod packings in

$$
L=\{(x, y, z): 0 \leq x+y+z \leq t\}
$$

for some given value of $t$, accepting those that are in canonical form under the action of $\langle r, p\rangle$. The accepted packings in $L$ are then extended to packings in $P$ before carrying out a final isomorph rejection (under the action of $\langle r, p\rangle$ ). The canonical form defined above respects approaches where packings are produced in such stepwise ways. Note that when carrying out isomorph rejection for a packing $C \subset P$, it suffices to consider group elements that are 
in the automorphism group of $C \cap L$. Consequently, if that group is trivial, then packings obtained can be accepted directly.

The entire search is completed by determining whether the accepted tripod packings in $P$ can be extended to packings of size $s$ by adding tripods from $R$.

\subsubsection{Odd $n$}

With odd $n,|Q|=n^{3}$ is odd, so for any partition $Q=P \cup R$, we have that $|P| \neq|R|$ and therefore $P \neq g R$ for all $g \in H$. Generalizations to partitions of $Q$ with more than two sets can be considered, but we have not found any natural such partitions either. Hence, we suggest a modification of the suggested approach for even $n$ and let

$$
\begin{aligned}
g & =o \\
P & =\{(x, y, z): 0 \leq x+y+z \leq 3 n / 2-5 / 2\} \\
M & =\{(x, y, z): x+y+z=3 n / 2-3 / 2\} \\
R & =\{(x, y, z): 3 n / 2-1 / 2 \leq x+y+z \leq 3 n-3\} .
\end{aligned}
$$

Now $P=o R$ and $M=o M$. The first step of the approach is to find all tripod packings in $M$, removing isomorphs under the action of $\langle r, p\rangle$. These act as starting points for the rest of the search, which considers words in $P$ directly without going via $L$. If a packing in $M$ has size $s^{\prime}$, then one only considers extensions in $P$ that have size at least $\left(s-s^{\prime}\right) / 2$. The canonical form requires a slight redefinition as $M$ has now overtaken the role of $L$ in the approach for even $n$. The final stage of completing the search is similar to that for even $n$.

\subsection{Results}

The described algorithms were implemented and computations were carried out on a compute cluster with 256 cores with 2.4-GHz Intel Xeon E5-2665 processors and 192 cores with 2.5-GHz Intel Xeon E5-2680v3 processors. A number of measures were taken to validate the results to be presented. Two different implementations of the algorithm were executed, and tables of intermediate data were produced. The tabulated results are useful for any later implementations and verifications. Double counting [4, Sect. 10.2] was used whenever possible - for example, for validating the number of packings in $L$ and $M$-and the programs were tested on smaller instances. 


\subsubsection{Case $n=10$}

For $n=10$ - the smallest open case - the described approach makes an exhaustive search feasible. Earlier it was known that $f(10) \geq 32$, so we search for tripod packings of size $s=33$. This is done by first finding, up to symmetry, all tripod packings of size at least $\lceil 33 / 2\rceil=17$ in $P$, the set of words $(x, y, z)$ with $x+y+z \leq 13$. We let $L=\{(x, y, z): 0 \leq x+y+z \leq 7\}$, and choose the condition $x+y+z \leq 7$ as the number of packings obtained after isomorph rejection, 56100, is suitable for distributing the computer search in the next step.

In $L$, there are 336133 tripod packings in total and 56100 packings up to symmetry. These tripod packings have between 1 and 9 tripods, and the number of packings of size 1 to 9 are 1, 23, 503, 5151, 19303, 23066, 7550, 499 , and 4 , respectively. The number of packings with an automorphism group of order 1,2,3, and 6 are 55959, 101, 36, and 4, respectively. The orbit-stabilizer theorem validates this part of the search, as $6 \cdot 55959+3$. $101+2 \cdot 36+4=336133$.

Data for the packings in $P$ are displayed in Table 2. Specifically, each tripod $C$ obtained in $P$, up to symmetry, adds 1 to the entry in row $|C \cap L|$ and column $|C|$.

\begin{tabular}{rrrr} 
Table 2: Number of packings in $P$ \\
\hline & 17 & 18 & 19 \\
\hline 1 & 0 & 0 & 0 \\
2 & 29 & 0 & 0 \\
3 & 93622 & 25 & 0 \\
4 & 12606901 & 17063 & 7 \\
5 & 155249767 & 574210 & 121 \\
6 & 313196630 & 1949479 & 1383 \\
7 & 113189132 & 903639 & 538 \\
8 & 7461674 & 80397 & 85 \\
9 & 26337 & 386 & 0 \\
\hline
\end{tabular}

Finally, data for the packings found in $Q$ are shown in Table 3, such that for each tripod $C$ accepted in $P$, a 1 is added to the entry in row $|C|$ and the column with the size of the largest extension of $C$ using tripods from $R$.

No packings were found of size 33 or greater, so $f(10)<33$ and thereby $f(10)=32$.

The computations took about 100 days of core time. 
Table 3: Number of packings in $Q$

\begin{tabular}{rrrrrr}
\hline & 25 & 26 & 27 & 28 \\
\hline 17 & 838 & 381942 & 13704021 & 131628563 \\
18 & 0 & 0 & 5135 & 177053 \\
19 & 0 & 0 & & 0 & 13 \\
\hline \multicolumn{6}{r}{} \\
& 29 & 30 & 31 & 32 \\
\hline 17 & 311661178 & 136516933 & 7909756 & 20861 \\
18 & 1267300 & 1798591 & 275578 & 1542 \\
19 & 307 & 1246 & 560 & 8 \\
\hline
\end{tabular}

\subsubsection{Case $n=11$}

For $n=11$ it was earlier known that $f(11) \geq 37$, but this bound is improved in Section 4 to $f(11) \geq 38$ so we shall here search for tripod packings of size $s=39$. We first find, up to symmetry under the action of $\langle r, p\rangle$, all tripod packings in $M$, the set of words $(x, y, z)$ with $x+y+z=15$. The number of such packings is 14639628 in total and 2440038 up to symmetry. Up to symmetry, the number of packings of size 0 to 11 are $1,18,515,8231$, 68920, 306847, 712894, 821580, 428684, 87260, 4664, and 424, respectively. The number of packings with an automorphism group of order 1, 2, 3, and 6 are 2439881, 30, 125, and 2, respectively, and the orbit-stabilizer theorem validates this part of the search: $6 \cdot 2439881+3 \cdot 30+2 \cdot 125+2=14639628$.

The accepted tripod packings in $M$ are now starting points for the rest of the search, which is carried out as described in Section 3.2.2. The hardest instances are those with the fewest tripods in $M$, and for parallelization purposes it makes sense to construct the packings in $P$ via packings in $L$, defined by (1), at least for packings with 0 and 1 tripods in $M$.

Data for the packings found in $Q$ are displayed in Table 4, such that for each tripod packing $C$ accepted in $P \cup M$, a 1 is added to the entry in row $(|C \cap M|,|C \cap P|)$ and the column given by the size of the largest extension of $C$ with tripods from $R$. Rows with only 0 entries are not included in the table (this happens for all cases with $8 \leq|C \cap M| \leq 11$ ).

No tripod packing was found of size 39 or greater, so $f(11)<39$ and thereby $f(11)=38$. There is nothing contradictory about the fact that not even a tripod packing of size 38 was found: later an optimal packing of size 38 will be presented which has $|C \cap M|=4$ and $|C \cap P|=|C \cap R|=17$; the case $(4,17)$ is not considered in Table 4 as $\left(s-s^{\prime}\right) / 2=(39-4) / 2>17$.

These computations took roughly 10 years of core time. 
Table 4: Number of packings in $Q$

\begin{tabular}{rrrrrrrrr}
\hline & 30 & 31 & 32 & 33 & 34 & 35 & 36 & 37 \\
\hline$(0,20)$ & 0 & 18 & 7675 & 414869 & 3841768 & 5328324 & 719746 & 5199 \\
$(0,21)$ & 0 & 0 & 0 & 8 & 331 & 1551 & 489 & 162 \\
$(1,19)$ & 76 & 20252 & 1828356 & 40324857 & 203028119 & 173629454 & 14214672 & 42529 \\
$(1,20)$ & 0 & 0 & 15 & 2853 & 64038 & 188161 & 58072 & 2246 \\
$(2,19)$ & 0 & 4 & 4820 & 266367 & 3010109 & 5654655 & 1084721 & 12529 \\
$(2,20)$ & 0 & 0 & 0 & 0 & 14 & 187 & 386 & 74 \\
$(3,18)$ & 0 & 3218 & 316067 & 7902084 & 50634637 & 58559271 & 6705056 & 28054 \\
$(3,19)$ & 0 & 0 & 0 & 98 & 2181 & 13621 & 10181 & 502 \\
$(4,18)$ & 0 & 0 & 20 & 3046 & 68133 & 220173 & 78678 & 1440 \\
$(5,17)$ & 0 & 0 & 1178 & 55109 & 600747 & 1138460 & 224643 & 1749 \\
$(5,18)$ & 0 & 0 & 0 & 0 & 16 & 60 & 30 & 0 \\
$(6,17)$ & 0 & 0 & 0 & 2 & 72 & 274 & 216 & 0 \\
$(7,16)$ & 0 & 0 & 0 & 0 & 68 & 474 & 332 & 0 \\
\hline
\end{tabular}

\section{Lower Bounds via Prescribed Symmetries}

If we search for a lower bound on $f(n)$ rather than the exact value, we may impose constraints on the tripod packings to reduce the search space. One option is to prescribe symmetries, that is, automorphisms, of the packings. Essentially, we are then searching for a set of orbits of codewords under the action of the prescribed automorphisms rather than a set of individual codewords. The codewords in Example 1 are partitioned into orbits under the action of $\langle r\rangle$.

\subsection{General Approach}

For the groups of automorphisms, we may restrict our consideration to one representative from each conjugacy class. The following result, stated without proof in [13, p. 1975], can be used to remove three of the classes from consideration.

Lemma 4.1. For $n \geq 3$, a code attaining $f(n)$ cannot have an automorphism that is a conjugate of $p$.

Proof. If there is a code that has an automorphism that is a conjugate of $p$, then there is a code $C$ of the same size that has $p$ as an automorphism. So if $(x, y, z) \in C$, then $(y, x, z) \in C$. If $x \neq y$, then the code does not satisfy (2) in the definition of a packing, so $x=y$ and all codewords are of the form $(x, x, z)$. Moreover, by (1) in the definition of a packing, if $(x, x, z)$ 
and $\left(x, x, z^{\prime}\right)$ are codewords, then $z=z^{\prime}$. Consequently the packing can have at most $n$ codewords, but we know that $f(n)>n$ when $n \geq 3$ - see [13] for lower bounds on $f(n)$ - so the code cannot attain $f(n)$.

By Lemma 4.1, we may ignore the groups (d), (g), and (i). This leaves the nontrivial groups (b), (c), (e), (f), and (h). The subgroup lattice containing these groups is presented in Figure 3. It is good to keep this lattice in mind: if $H_{1}<H_{2}$ and $H_{1}$ is prescribed, then the largest tripod packing is at least as large as the largest packing when $H_{2}$ is prescribed.

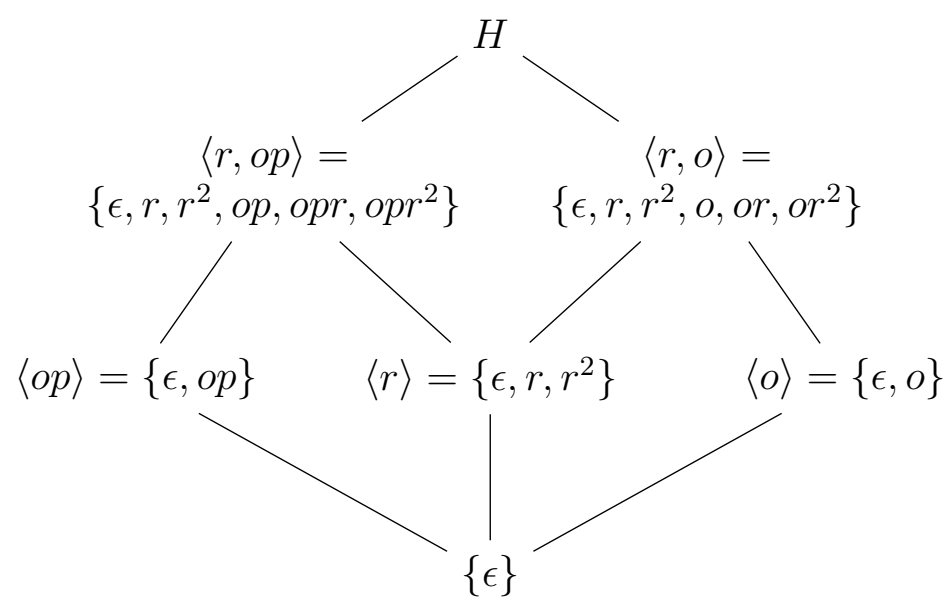

Figure 3: Lattice of subgroups considered

When prescribing a group $H^{\prime}$, the symmetries of the search space are captured in the normalizer of $H^{\prime}$ in $H$, where the normalizer is defined as $\mathrm{N}_{H}\left(H^{\prime}\right)=\left\{h \in H: h H^{\prime}=H^{\prime} h\right\}$. The normalizer $\mathrm{N}_{H}\left(H^{\prime}\right)$ is partitioned by cosets of $H^{\prime}$. Relevant remaining symmetries are essentially about the quotient group $\mathrm{N}_{H}\left(H^{\prime}\right) / H$, which one can deal with through one representative from each coset.

For the subgroups $\langle r\rangle,\langle o\rangle,\langle r, o\rangle$ and $\langle r, o p\rangle$ the normalizer is the whole group $H$, and for $\langle o p\rangle$ we have $\mathrm{N}_{H}(\langle o p\rangle)=\{\epsilon, o, p, o p\}$. In particular, the element $o$ is in the normalizer of $\langle r\rangle$, so when prescribing the group (b), the approach of dividing $Q$ into two parts, $P$ and $R$, as discussed in Section 3.2 is applicable also here.

In the basic case, when $Q$ is not divided into two parts, we arrive at instances of the maximum weight clique problem, where the graph is obtained in the following way. Consider the action of the prescribed group; this action gives orbits of codewords. There is a vertex for each such orbit whose 
codewords satisfy the distance conditions (1) and (2) pairwise. The weight of a vertex is the length of the corresponding orbit. There is further an edge between two vertices when the conditions (1) are (2) are satisfied for all pairs $(c, d)$ of codewords, where $c$ is taken from one of the orbits and $d$ from the other. Instances of the maximum weight clique problem can also be handled by Cliquer.

\subsection{Results}

Table 5 shows the size of the largest tripod packings for each of the five groups considered. These packings imply lower bounds on $f(n)$. The bounds $f(11) \geq 38$ and $f(16) \geq 68$ are new, improving on the previous bounds $f(11) \geq 37$ and $f(16) \geq 65$ from [13]. Now, the exact values for all entries in [13, Table 1] are known. See also Table 1, where we further extend the range of bounds considered in earlier studies by including the cases $17 \leq n \leq 26$. The new packings for $n=11$ and $n=16$ are displayed as monotonic matrices in Figures 4 and 5, respectively. These two packings as well as packings corresponding to all other entries in Table 5 can be obtained electronically from $[8]$.

The following conjecture is stated in [13].

Conjecture 4.1 (3-symmetry). For every $n$, there exists a tripod packing attaining $f(n)$ with $\langle r\rangle$ as a group of automorphisms.

The results for $n=11$ disprove Conjecture 4.1: the largest packing for $\langle r\rangle$ has size 37, whereas the largest packing for $\langle o p\rangle$ has size 38. We refrain from making any further conjectures regarding automorphisms of optimal tripod packings. Extremal combinatorial structures tend to have nontrivial symmetries, but here the situation is somewhat atypical as the order of the group $H$ is only 12 .

\section{Acknowledgments}

The authors would like to thank Sándor Szabó for encouraging them to work on tripod packings.

\section{References}

[1] R. Ahlswede, H. Aydinian, and L. H. Khachatrian, Unidirectional error control codes and related combinatorial problems, in: Proc. 8th 
Table 5: Tripod packings with prescribed symmetries

\begin{tabular}{cccccc}
\hline$n \backslash$ Group & $\langle r\rangle$ & $\langle o\rangle$ & $\langle r, o\rangle$ & $\langle o p\rangle$ & $\langle r, o p\rangle$ \\
\hline 3 & 5 & 3 & 3 & 5 & 5 \\
4 & 8 & 8 & 8 & 8 & 4 \\
5 & 11 & 9 & 9 & 11 & 11 \\
6 & 14 & 14 & 14 & 14 & 14 \\
7 & 19 & 17 & 17 & 19 & 19 \\
8 & 23 & 22 & 22 & 22 & 22 \\
9 & 28 & 27 & 27 & 28 & 28 \\
10 & 32 & 32 & 32 & 32 & 30 \\
11 & 37 & 37 & 35 & 38 & 37 \\
12 & 43 & 42 & 42 & 42 & 42 \\
13 & 49 & 47 & 47 & & 47 \\
14 & 55 & & 54 & & 54 \\
15 & 61 & & 59 & & 61 \\
16 & 68 & & 66 & & 66 \\
17 & & & 73 & & 75 \\
18 & & & 80 & & 80 \\
19 & & & 87 & & 87 \\
20 & & & 94 & & 94 \\
21 & & & 101 & & 104 \\
22 & & & 110 & & 110 \\
23 & & & 121 & & 119 \\
24 & & & 124 & & 126 \\
25 & & & & & 146 \\
26 & & & &
\end{tabular}




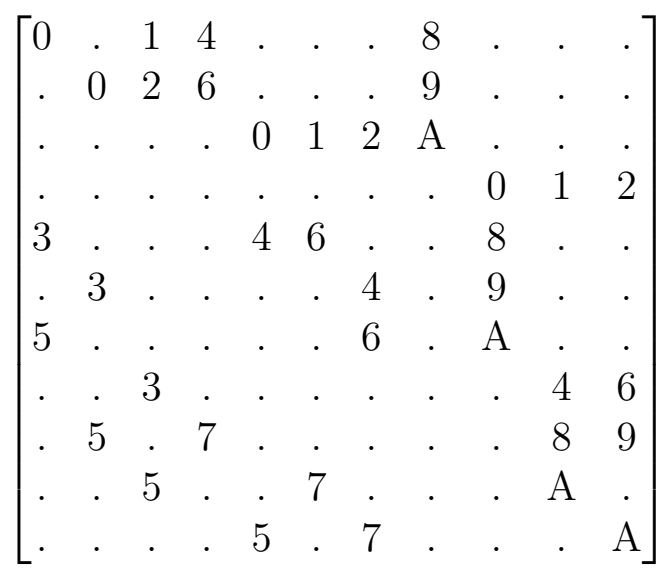

Figure 4: Monotonic matrix showing $f(11) \geq 38$



Figure 5: Monotonic matrix showing $f(16) \geq 68$ 
Int'l Workshop on Algebraic and Combinatorial Coding Theory (Tsarskoe Selo, Russia, September 8-14, 2002), pp. 6-9.

[2] S. W. Golomb, A general formulation of error metrics, IEEE Trans. Inform. Theory 15 (1969), 425-426.

[3] W. Hamaker and S. Stein, Combinatorial packing of $\mathbb{R}^{3}$ by certain error spheres, IEEE Trans. Inform. Theory, 30 (1984), 364-368.

[4] P. Kaski and P. R. J. Östergård, Classification Algorithms for Codes and Designs, Springer, Berlin, 2006.

[5] T. Kløve, J. Luo, and S. Yari, Codes correcting single errors of limited magnitude, IEEE Trans. Inform. Theory 58 (2012), 2206-2219.

[6] S. Niskanen and P. R. J. Östergård, Cliquer User's Guide: Version 1.0, Technical report T48, Communications Laboratory, Helsinki University of Technology, Espoo, 2003.

[7] P. R. J. Östergård, A fast algorithm for the maximum clique problem, Discrete Appl. Math. 120 (2002), 197-207.

[8] P. R. J. Östergård and A. Pöllänen, Dataset for New results on tripod packings [Dataset]. Zenodo. http://doi.org/10.5281/zenodo.1230276 (April 26, 2018).

[9] S. K. Stein, Factoring by subsets, Pacific J. Math. 22 (1967), 523-541.

[10] S. Stein, Packings of $\mathbb{R}^{n}$ by certain error spheres, IEEE Trans. Inform. Theory, 30 (1984), 356-363.

[11] S. K. Stein and S. Szabó, Algebra and Tiling: Homomorphisms in the Service of Geometry, Mathematical Association of America, Washington, 1994.

[12] S. Szabó, Monotonic matrices and clique search in graphs, Ann. Univ. Sci. Budapest. Sect. Comput. 41 (2013), 307-322.

[13] A. Tiskin, Packing tripods: narrowing the density gap, Discrete Math. 307 (2007), 1973-1981. 